\title{
Prevalence of Urinary Schistosomiasis among School Aged Children in Khelawa Village, River Nile State, Sudan, 2017
}

\begin{abstract}
Mosab Nouraldein Mohammed Hamad*, Esraa Abid Said Ahmed, HalaAlaa Hassan Omer, Duaa Abd Elrahman Elhaj, Arwa Fathi Mahmoud, Omer Hashim Abd Elsalam, Tasneem Khalid Ahmed, AlaaAlkhair Abo Elgasem and Tagwa Ahmed Mahmoud
\end{abstract}

Faculty of Health Sciences, Elsheikh Abdallah Elbadri University, Sudan

*Corresponding author: Mosab Nouraldein Mohammed Hamad, Parasitology and Medical Entomology Department, Medical Laboratory Sciences Department, Faculty of Health Sciences, Sudan

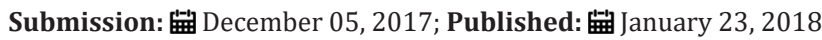

\begin{abstract}
Background: Urinary schistosomiasis is a disease caused by parasitic worms which, if left untreated, can eventually lead to anaemia, malnutrition, kidney failure, or bladder cancer. The disease occurs mainly in school-aged children and young adults in sub-Saharan Africa.

Justification: Schistosomiasis is one of the most parasitic diseases with serious complications that may lead to infertility, renal failure and bladder carcinoma.
\end{abstract}

Objectives: To know the prevalence of Schistosoma hematobium among school aged children in Khelawa village, River Nile State, Sudan.

Materials and method: Descriptive, cross sectional study, 200 urine specimens were collected from each participant and examined by parasitological method.

Result: $32.5 \%$ of school aged children were infected with Schistosoma haematobium.

Conclusion: High percentage of urinary schistosomiasis among study group is attributed to patient's behaviors, presence of irrigated scheme near their inhabitants and due to absence of qualified health care unit.

Recommendations: Further studies are recommended applying more diagnostic methods and including large number of population

Keywords: Urinary schistosomiasis; School aged children

\section{Introduction}

Urinary schistosomiasis is a disease caused by parasitic worms which, if left untreated, can eventually lead to anaemia, malnutrition, kidney failure, or bladder cancer. The disease occurs mainly in school-aged children and young adults in sub-Saharan Africa [1].

The parasite is found in the venous plexus draining the urinary bladder of humans. During infection, the parasites deposit terminal spined eggs which clog the venous plexus, impeding blood flow. This bursts the veins, allowing blood and eggs to enter the urinary bladder, resulting in the characteristic symptom of blood in urine or haematuria. In sub-Saharan Africa alone it is estimated that 70 million individuals experience heamaturia, 32 million with difficulty in urinating (dysuria), 18 million with bladder-wall pathology, and 10 million with major hydronephrosis from infection caused by Schistosoma haematobium. Mortality rate due to nonfunctioning kidney (from S. haematobium) and haematemesis has been put at 150,000 per year. The above figures imply that urinary schistosomiasis is an important public health problem in subSaharan Africa and second to malaria in morbidity [2].

In Brazil and Africa, refugee movements and migration to urban areas are introducing the disease to new locations. Increasing population size and corresponding needs for power and water have led to increased transmission. Infections are not uniformly distributed within communities. It has been estimated that $5-10 \%$ of an endemic community may be heavily infected, and the remainder has mild to moderate infections. The risk of infection is highest amongst those who lived near lakes or rivers. In Uganda, almost no transmission was found to have occurred at altitudes greater than $1400 \mathrm{~m}$ or where the annual rainfall was less than 900 $\mathrm{mm}$. With the rise of tourism and travel, an increasing number of tourists are contracting it. Tourists often present with severe acute infection and unusual problems including paralysis [3]. 
In this review, the clinical manifestations of urinary schistosomiasis are displayed from a pathogenetic perspective. According to the prevailing host's immune response profile, urinary schistosomiasis may be broadly categorized into cell-mediated and immune-complex-mediated disorders. The former, usually due to Schistosoma haematobium infection, are attributed to the formation of granulomata along the entire urinary tract. As they heal with excessive fibrosis, they may lead to strictures, calcifications and urodynamic abnormalities. The main impact is lower urinary, the site of heaviest ovi-position. Secondary bacterial or viral infection is common, any may be incriminated in secondary stone formation of the development of bladder malignancy. Immune-complex mediated lesions are usually associated with hepatosplenic schistosomiasis due to Schistosoma mansoni infection. Circulating complexes composed of schistosomal gut antigens and different classes of immunoglobulins deposit in the kidneys leading to several patterns of glomerular pathology. The latter have been categorized under six classes based on the histological and immune fluorescence profile. These classes have been linked to respective clinical manifestations and depend on the stage of evolution of the host's immune response, extent of associated hepatic fibrosis and co-infection with salmonella or hepatitis C. Secondary amyloidosis develops in $15 \%$ of such patients, representing a critical impairment of macrophage function.

\section{Conclusion}

The wide clinic pathological spectrum of urinary schistosomiasis mirrors the evolution of the host's immune response according to chronicity of infection, bacterial or viral co-infection and, in the case of glomerulonephritis, to the extent of hepatic co-morbidity [4].

The classic sign of urogenital schistosomiasis is haematuria (blood in urine). Fibrosis of the bladder and ureter, and kidney damage are sometimes diagnosed in advanced cases. Bladder cancer is another possible complication in the later stages. In women, urogenital schistosomiasis may present with genital lesions, vaginal bleeding, and pain during sexual intercourse, and nodules in the vulva. In men, urogenital schistosomiasis can induce pathology of the seminal vesicles, prostate, and other organs. This disease may also have other long-term irreversible consequences, including infertility.

The economic and health effects of schistosomiasis are considerable and the disease disables more than it kills. In children, schistosomiasis can cause anaemia, stunting and a reduced ability to learn, although the effects are usually reversible with treatment. Chronic schistosomiasis may affect people's ability to work and in some cases can result in death. The number of deaths due to schistosomiasis is difficult to estimate because of hidden pathologies such as liver and kidney failure and bladder cancer [5]

\section{Justifications}

Schistosomiasis is one of the most parasitic diseases with serious complications that may lead to infertility, renal failure and bladder carcinoma.

\section{Objectives}

To know the prevalence of Schistosoma hematobium among school aged children in Khelawa village, River Nile State, Sudan.

\section{Material and Methods}

\section{Study design}

Descriptive, cross sectional study

\section{Study area}

Khelawa village, River Nile State, Sudan

\section{Study population}

School aged children in Khelawa village, River Nile State, Sudan

\section{Sample size}

200 samples

\section{Ethical consideration}

The participants were informed about the goal of the study and all of them were consent to participate in the study.

\section{Methods}

\section{Specimen type}

Urine

\section{Diagnostic method}

Parasitological method, examination of urine deposit for Schistosoma haematobium ovum.

\section{Result}

$32.5 \%$ of school aged children were infected with Schistosoma haematobium.

\section{Conclusion}

High percentage of urinary schistosomiasis among study group is attributed to patient's behaviors, presence of irrigated scheme near their inhabitants and due to absence of qualified health care unit.

\section{Recommendations}

Further studies are recommended applying more diagnostic methods and including large number of population.

\section{References}

1. http://www.who.int/tdr/news/2014/urinary-schisto-treatmt/en/

2. Uwem FE, Laja-Deile A, Oluwole AS, Sam-Wobo SO, Mafiana CF, et al. (2010) Urinary schistosomiasis among preschool children in a rural community near Abeokuta, Nigeria. BMC Parasites \& Vectors 3: 58.

3. https://emedicine.medscape.com/article/228392-overview

4. Barsoum RS (2013) Urinary Schistosomiasis: Review. Journal of Advanced Research 4(5): 453-459.

5. http://www.who.int/mediacentre/factsheets/fs115/en 